\title{
Cuba, par-delà les océans : un film sur la possession
}

Cuba, Beyond the Oceans: A Film about the Possession

\section{Caterina Pasqualino}

\section{OpenEdition}

Journals

Édition électronique

URL : http://journals.openedition.org/recherchestravaux/1171

DOI : 10.4000/recherchestravaux.1171

ISSN : 1969-6434

\section{Éditeur}

UGA Éditions/Université Grenoble Alpes

\section{Édition imprimée}

ISBN : 978-2-37747-065-5

ISSN : 0151-1874

\section{Référence électronique}

Caterina Pasqualino, "Cuba, par-delà les océans : un film sur la possession », Recherches \& Travaux [En ligne], 93 | 2018, mis en ligne le 26 octobre 2018, consulté le 08 septembre 2020. URL : http://

journals.openedition.org/recherchestravaux/1171; DOI : https://doi.org/10.4000/recherchestravaux. 1171

Ce document a été généré automatiquement le 8 septembre 2020.

(c) Recherches \& Travaux 


\title{
Cuba, par-delà les océans : un film sur la possession
}

\author{
Cuba, Beyond the Oceans: A Film about the Possession
}

Caterina Pasqualino

1 Yamilka souffre profondément de l'absence de son fils Odin qui lui a été enlevé à l'âge de neuf ans par son mari parti émigrer à Toulouse. Pour soulager sa douleur, elle communique avec les esprits des morts qu'elle a hérités de sa mère. Son préféré est celui qu'elle nomme Negro bruto, l'esprit d'un défunt qui, de son vivant, répondait au nom de Juaquim. Elle affirme pouvoir préserver la relation avec son fils absent, qui a aujourd'hui une vingtaine d'années, par l'intermédiaire d'une poupée de tissu qui représente Negro bruto, d'un récipient contenant divers objets de culte ainsi qu'à travers ses rêves et ses visions ${ }^{1}$. Les morts venant posséder Yamilka sont ses « fantômes de la mémoire ». Dans le film, j'explore les circonstances dans lesquelles ils viennent la hanter. Dans cet univers très secret, la présence de la caméra s'est paradoxalement révélée être une aide précieuse: malgré l'artifice de la mise en scène, à plusieurs reprises elle suscita leurs venues.

2 J'avais déjà travaillé à Cuba en 2007, 2008 et 2011 sur le comportement de possédés chevauchés par des morts. Leurs transformations physiques sont spectaculaires. Ils adoptent une déambulation stéréotypée, machinale, comme s'ils perdaient leur humanité pour devenir une "chose», une "materia». Une fois réduits à cet état d'hommes-objets, ils sont censés être capables de dialoguer avec d'autres objets situés dans leurs alentours. Selon Philippe Descola, l'animisme implique qu'au-delà de leur différence constitutive, une pierre et un homme partagent "une même sensibilité ${ }^{2}$. Dans ce système, non seulement la pierre est dotée d'affects, mais certaines forces spirituelles circulent entre elle et l'homme. Tim Ingold et Eduardo Viveiros de Castro ont suggéré qu'une telle conception animiste peut se retrouver dans différentes sociétés. Dans l'idée de repenser l'expérience de "chosification» comme une expérience contemporaine, j'ai proposé ailleurs une comparaison entre la transe palo et le théâtre de Thadeus Kantor. 
3 Je suis retournée six ans plus tard, en novembre 2017, pour retrouver Yamilka, une palera qui avait attiré mon attention dès mon premier séjour. Belle, vive et exubérante, elle est toujours en mouvement, ses yeux expressifs semblant constamment interroger son entourage. Curieuse, elle ne se lasse pas de partir à la recherche de tout ce qui peut lui être utile pour ses rituels. Dans son mudanzo, la pièce qu'elle réserve chez elle à ses pratiques religieuses, elle rassemble les objets les plus insolites. Le but est de soigner les membres de sa famille, ses voisins, mais aussi des inconnus devenus des " patients".

4 Voici donc des années que nous nous connaissons et sommes liées par une affinité reposant sur notre proximité d'âge et par des liens d'amitié. Malgré cela, son sourire ambivalent me met parfois dans l'embarras. Derrière la femme " entière » qu'elle aime incarner, elle reste secrète. Mon désir de retourner la voir était motivé par son allure mystérieuse composée d'un mélange de soumission, de résistance et d'invention que Michel Foucault aurait compté parmi les «Vies des hommes Infâmes ${ }^{3}$ ». Dans ce texte, Foucault souligne notamment le rôle que la poésie et la littérature ont à jouer pour dire l'intensité d'expériences d'hommes et de femmes humbles. Mon souvenir de Yamilka était si intense qu'une fois sur place je fus prise par un doute : et si je venais à Cuba pour tenter de me libérer de sa présence obsédante?

Lorsque nous nous retrouvons, nous nous saluons par une étreinte chaleureuse. Yamilka m'annonce aussitôt qu'elle est devenue grand-mère. Je la filme radieuse, dans un moment de grâce. Ma petite fille est née il y a 45 heures me dit-elle, à Toulouse, en France. Je contacte aussitôt son fils Odin qui est l'heureux papa pour lui passer Yamilka (la veille, elle avait déjà pu parler à son fils par téléphone). Cette dernière lui demande de décrire le nouveau-né, une petite-fille. Elle lui demande de lui dire que sa grandmère l'aime. À ce moment précis, Yamilka prend subitement une expression contrariée et douloureuse. La joie de cette naissance est mêlée de regrets, de révolte, de ressentiment et de jalousie. Son fils Odin a aujourd'hui vingt et un ans. En douze ans, il n'est venu à Cuba qu'à trois reprises, et encore, chaque fois c'était pour un court séjour.

\section{Yamilka, Leonardo et la Française}

6 Lorsqu'il était jeune, son mari Leonardo était si beau que toutes ses amies l'enviaient. Ils eurent un enfant, Odin, et vécurent ensemble pendant une dizaine d'années, très pauvres, mais heureux. Jusqu'au jour où une touriste française s'éprit de Leonardo. Ce dernier expliqua tout d'abord à Yamilka que ce flirt n'était pas important et que, comme l'étrangère le payait, cela leur permettait au moins d'assurer provisoirement leur subsistance ainsi que celle de leur fils Odin. Il la convainquit que, sur l'île, beaucoup auraient rêvé d'une telle opportunité. Yamilka se résolut à accepter momentanément cette liaison extraconjugale. La relation avec son mari se normalisa au point qu'il leur arrivait de se moquer de ces Européens immatures venant à Cuba « pour recharger leurs batteries " en cherchant à éprouver des sensations fortes. Et puis l'argent de la Française les sortait de la misère, tandis que cette dernière ne se doutait pas que Leonardo était marié...

7 Mais l'étrangère revint régulièrement. Leonardo, qui, pendant les premiers séjours, s'éclipsait de son foyer pendant quelques jours, finit par s'absenter plus longtemps, à savoir pendant tout le mois que duraient les vacances de la touriste. Odin, âgé de deux ans, commençait déjà à trottiner, pieds nus dans la boue de leur bidonville dès que quelques gouttes de pluie tombaient. Leonardo revenait régulièrement avec l'argent 
qui les nourrissait tous trois. Une fois retournée chez elle, la Française commença à envoyer de temps à autre de petites sommes. Ils s'y habituèrent jusqu'à en devenir dépendants : combien de fois avaient-ils attendu cette manne providentielle...

Si Yamilka savait que cette situation ne durerait pas, elle n'imaginait cependant pas la tournure dramatique qu'allait prendre cette aventure. Lorsque l'État commença à autoriser les Cubains à fréquenter les grands établissements touristiques, Leonardo put fréquenter de luxueux hôtels pour rejoindre sa Française. C'est ainsi qu'il commença peu à peu à envisager de quitter Cuba où il menait une vie misérable et sans aucune perspective d'avenir. S'imaginant une vie dorée en France, il se prit à rêver de Toulouse.

Quelques années auparavant, de nombreux Cubains tentaient sans relâche de fuir la misère en s'embarquant au péril de leur vie sur de frêles barques pour tenter de franchir la bande de mer qui les séparait de Miami. Mais cette échappatoire a disparu. Les voyages clandestins s'étaient interrompus dès lors que les États-Unis ont refusé le droit d'asile aux réfugiés cubains. Quelques-uns ont bien continué à tenter leur chance en construisant secrètement des bateaux malgré leur crainte d'être signalé par un voisin à la police d'État. Mais les énormes difficultés pour s'en sortir par ce biais tarirent la filière. Le moyen le plus sûr de quitter l'île était désormais de se marier avec un étranger ou une étrangère.

Leonardo supportait d'autant moins la misère de son quartier que son désir était excité par tout ce que la Française lui envoyait : denrées rares, jeans, chemises... Yamilka et Leonardo vivaient au rythme des allers et retours de la Française. Un beau jour, Leonardo annonça à Yamilka qu'elle voulait l'épouser. Sa joie était visible. Son rêve allait enfin se réaliser. Il lui expliqua qu'il le faisait pour elle et pour Odin, et qu'il lui enverrait de l'argent. Elle fut d'abord tétanisée par la nouvelle. Mais sous la pression de Leonardo, elle se résolut peu après à signer l'indispensable procédure de divorce. Après avoir épousé la touriste, Leonardo obtient le fameux visa d'expatriation pour la France... Pour Yamilka, une chose était de supporter une trahison dont elle s'arrangeait le temps des vacances de la Française, une autre était d'accepter le départ irréversible de son mari et d'assumer son sentiment d'abandon. Pire, alors qu'elle était dans un état de grande faiblesse psychologique, Yamilka accepta l'inacceptable. Sous les suppliques de Leonardo et les conseils de sa propre mère, elle laissa son fils Odin, alors âgé de neuf ans, le rejoindre à Toulouse. Sa mère la consola en affirmant que cette séparation était certainement voulue par l'esprit Negro bruto et qu'elle ne devait pas s'en inquiéter. Tôt ou tard, son sacrifice serait payé en retour en la sortant de la misère et par la reconnaissance éperdue de son fils Odin.

\section{Filmer l'intime}

11 Je suis actuellement en train de monter les premières scènes du film. La plupart des scènes ont pour cadre son appartement. La première se situe dans la pièce (mudanzo) qu'elle réserve au culte des esprits défunts. Assise sur une chaise, elle s'adresse à celui qui lui est le plus proche: Negro bruto.

Negro bruto est un ancêtre africain réduit en esclavage à Cuba. Il est décrit comme sentant mauvais, petit et se déplaçant en traînant les pieds car ses chevilles sont entravées par des chaînes. L'effigie en tissu qui le représente est de confection rustique. Son visage est noir et rond, deux coquillages font office d'yeux. Comme au temps de 
l'esclavage à la campagne, il est vêtu d'une chemise blanche et d'un chapeau de paille. Quant au récipient dans lequel il vit, il se présente comme un microcosme composé d'éléments humains (fragments de crânes, divers ossements ou cheveux), d'éléments provenant du monde naturel (bâtons incorporés dans des mottes de terre, fragments de bois, coquillages, herbes et pierres ramassées sous la mer ou dans le lit d'une rivière), de matériaux (cordes, diverses ferrailles, barres de fer, clous, chaînes), et d'éléments manufacturés (croix, poupée, figurine en bois, vieille chaussure, avion miniature et divers objets brisés). Negro bruto assiste Yamilka dans toutes ses tâches cultuelles. Il est prêt à se bagarrer pour elle, mais il est aussi jaloux qu'un amant.

Dans une séquence filmée, Yamilka s'adresse à lui d'une voix calme et péremptoire. Elle lui rappelle qu'elle cherche à le satisfaire le mieux qu'elle peut en lui soufflant dessus des nuées de rhum. En ce moment elle ne peut malheureusement pas lui fournir de tabac parce qu'elle n'en a pas les moyens, mais le lendemain elle lui offrira des herbes sauvages. Yamilka sait qu'elle peut compter sur lui et sur tous les morts qui résident dans sa maison, non seulement pour l'aider à résoudre les situations délicates de ses patients, mais aussi pour protéger son fils.

Je la filme en plan très rapproché. À quelques centimètres du visage, je parviens mieux à saisir ses expressions faciales exacerbées par le contre-jour et la lumière rasante. Yamilka revient sur la trahison et l'abandon de Leonardo. Nimbée de doré et de rose, la scène semble se dérouler comme dans un rêve. Tout en s'adressant à Negro bruto, Yamilka résume brièvement sa situation, rappelant lentement et sur un ton détaché qu'elle conserve ce drame encore très présent dans sa mémoire. Égrenant ses paroles, se remémorant à haute voix les promesses non tenues par Leonardo, elle dit sa confiance qu'un jour il finira par comprendre d'où vient véritablement son fils et qui est sa vraie mère. «La loi de Dieu ne peut être bafouée impunément : - En marche Negro Bruto, fais-le-lui savoir ! » s'exclame-t-elle.

La séquence suivante est tournée dans le salon. Installée dans un canapé, Yamilka y revit sa douleur. Elle ne s'adresse plus à un public imaginaire, elle s'apitoie sur le sort que lui a réservé la vie en versant des larmes. Elle confie que son fils Odin lui apparaît régulièrement en pleine nuit comme s'il était mort. Elle se réveille alors en sueur et le voit se dresser devant elle. Les milliers de kilomètres qui les séparent sont comme annulés. Elle pressent qu'à Toulouse son fils souffre. Lorsqu'elle se réveille, elle s'écrie : «Negro Bruto qu'est-ce qui arrive à Odin ? Il ne va pas bien!» Comme les mois s'écoulent sans qu'elle puisse étreindre son fils, elle intensifie ses relations avec ses morts pour les interroger. Pour répondre à ses questions, Negro bruto lui demande toujours plus de compensations.

16 «Odin, téléphone-moi!» s'exclame Yamilka entre deux sanglots. Elle évoque ce dialogue imaginaire: "Maman, est censé répondre son fils, je ne veux pas que tu apprennes ce qui m'arrive pour ne pas te peiner... » Et elle lui répondrait : « Tu dois tout me dire parce que je suis ta mère! » Puis elle l'interpelle comme elle le fait avec Negro bruto: "Tu es venu me voir, mon fils! Je sens que les voix des morts le chantent ! » Elle entend alors la voix de son fils lui demander de se laver à l'aide de décoctions de plantes médicinales pour se calmer, se reposer, pour être belle et arrêter de trembler...

17 Cette séquence à forte tension émotionnelle a été tournée sur un mode lent et fluide, elle rompt avec le style habituellement enlevé des reportages documentaires. 
18 À partir de ce moment, la narration filmique se fait plus intense, plus empathique. Jusqu'à présent, Yamilka se retranchait dans son rôle public de prêtresse tandis qu'elle me reléguait au rôle de simple caméraman. Pour échapper à l'antagonisme filmeur / filmé, nous allons maintenant tenter d'expérimenter une certaine désorientation et plus d'incohérence.

19 Afin de nous éloigner des bruits d'un quartier trop bruyant, nous nous installons dans l'intimité de la chambre de Yamilka. La pièce est petite, presque entièrement occupée par le lit matrimonial. Comme il y a peu de place, elle se trouve naturellement assise au bord du lit, dos au mur. Elle se détend peu à peu. Elle souhaite revenir sur le fait qu'elle est née avec une maladie cardiaque. Âgée de quelques mois seulement, les médecins l'avaient déclarée cliniquement morte. Ses funérailles annoncées, son corps avait été allongé dans un petit cercueil. C'est à ce moment qu'un de ses oncles se rendit compte qu'un miroir installé à l'intérieur du cercueil s'était couvert de buée, preuve que Yamilka respirait à nouveau! Après cette "résurrection », Yamilka vécut pendant des années comme en sursis, les médecins ayant prédit qu'elle ne survivrait de toute façon pas au-delà de ses quinze ans. C'est sans doute consciente d'avoir à combattre sa maladie que dès l'âge de cinq ans ses rêves commencèrent à être hantés par Negro bruto. Celui-ci ne cessa de prendre de l'importance au fil des ans. Il était le signe que, comme sa mère, Yamilka était destinée à devenir mortera, c'est-à-dire une prêtresse ayant un don de médiation avec les ancêtres défunts pour résoudre les problèmes rencontrés par son entourage.

\section{Une histoire d'intimité}

Yamilka évoque son histoire miraculeuse sur un ton détaché, tout en accrochant au mur une photo d'elle à l'âge de cinq ans. Dans cette famille très pauvre, aucun de ses frères n'a eu la chance de se faire photographier enfant. Sa mère l'avait emmenée chez un photographe professionnel seulement parce que, condamnée par les médecins, elle voulait conserver un souvenir après la mort de sa fille. En contemplant la photo ancienne accrochée au-dessus de son lit, Yamilka sourit, émue. Je filme ce moment d'introspection, dialogue entre les deux Yamilka, la petite fille fragile et la belle et robuste femme qu'elle est devenue aujourd'hui. Souvenirs d'un passé dramatique et ambivalent : d'un côté Yamilka déteste sa mère et Negro bruto parce qu'elle les rend responsables du départ de son fils; de l'autre elle les adore parce qu'ils la protègent en la maintenant en vie dans un équilibre entre la réalité de ce monde et l'au-delà, entre la vie et la mort.

21 La séquence de Yamilka contemplant la photo d'elle enfant marque un basculement du scénario. À ce moment, elle passe doucement d'une position assise à une position allongée. Puis elle fait mine de dormir et change de registre. Quelques jours plus tôt, je lui avais demandé de collaborer à l'écriture du film et à la mise en scène ${ }^{4}$. Elle ne m'avait pas répondu sans doute parce qu'elle n'en voyait pas l'intérêt. Mais sa position allongée change la donne. C'est elle qui décide à présent de l'évolution du film ${ }^{5}$. Elle se livre à des confidences beaucoup plus intimes. Elle évoque la jalousie de Negro bruto arrivant jusqu'à s'endormir entre elle et son nouveau mari Norberto... Comme ce dernier craint la fureur de Negro bruto, il encourage prudemment Yamilka à se soumettre totalement à lui. 
22 Negro bruto est aussi jaloux des autres morts résidants dans la maison. Il ne supporte pas qu'elle se laisse posséder («que tenga otros cavallos ») par d'autres morts, surtout par Sarabanda. Il en devient menaçant, exigeant un amour exclusif, et devient parfois présomptueux. Estimant qu'il vaut plus que tous les autres morts, il réclame plus de considération. Récemment, il a déclaré ne plus accepter de vivre dans un récipient de petite taille et qu'il voulait être installé dans un récipient au moins aussi grand que celui de Sarabanda, un des morts les plus importants du Palo monte $e^{6}$.

\section{Ce qu'être possédé veut dire}

23 Après s'être assurée que je suis prête à filmer, Yamilka me prévient qu'elle va me raconter les moments où Negro bruto vient l'avertir des complots ourdis contre son fils en France.

24 C'est lorsqu'elle se trouve dans un état de sommeil éveillé qu'elle se souvient le mieux de l'histoire cruelle de l'abandon de son mari, du divorce qui suivit, et surtout du départ de son enfant aimé. Elle m'explique maintenant comment elle passe d'un état de somnolence à la transe, ce qui peut prendre une tournure assez effrayante. Lorsqu'elle va être possédée par Negro bruto, elle pleure, sanglote, vocifère ou murmure. Sa voix se brise, elle émet des sons rauques, lents, graves, comme si elle était distante et parlait depuis l'au-delà. Elle semble alors incarner Negro bruto en personne, adoptant une langue étrange, incompréhensible, censée n'appartenir qu'à lui.

25 Si elle le voit et lui parle quasi quotidiennement, elle se laisse posséder de préférence dans les occasions festives, par exemple la veille des célébrations de San Lazaro et de Santa Barbara. Lorsque l'émotion est forte, Negro bruto ne lui laisse aucun répit ${ }^{7}$. Il lui envoie des sortes de décharges électriques qui l'immobilisent, la laissant épuisée plusieurs jours de suite ${ }^{8}$.

26 Au cours de mes enquêtes sur le Palo monte à Santiago de Cuba, j'ai découvert la notion de ache. Elle peut être entendue comme un phénomène qui déclenche chez les possédés une montée d'émotion s'exprimant par des frissons. Variantes du célèbre concept polynésien du mana étudié par Mauss, le ache marque une phase d'intensification du rituel. Le ache se manifeste lors d'un changement de rythme, lors du solo intempestif d'un participant ou lors d'un mouvement inattendu et brusque du possédé : soit lors d'un "tourbillon dangereux» nommé cruce (dont la traduction littérale est " croisement»). Ces signaux marquent l'arrivée d'un mort venu chasser violemment un autre mort pour s'emparer du corps d'un possédé.

Yamilka affirme que dans ces moments les barrières temporelles et spatiales n'existent plus. Negro bruto serait fulgurant, bien plus rapide qu'un avion, que la vitesse de la lumière ou que la transmission d'une émotion. Il se transporterait souvent pour elle audelà des océans et reviendrait aussi vite pour l'informer de la santé de son fils et de la vie qu'il mène en France. Se lier à Negro bruto lui permettrait d'échanger avec son fils vivant à des milliers de kilomètres. Elle parviendrait même à éprouver la sensation de vivre en sa compagnie pendant des jours.

28 Au lieu d'avoir émigré comme son ex-mari à la recherche d'un monde plus clément, Yamilka s'évade de la misère par l'esprit. Il y a quelques années, j'avais interprété ce comportement comme l'expression d'une marginalité revendiquée contre une société cubaine sous le joug de la police et de l'armée. Pauvres parmi les plus pauvres, les adeptes du Palo monte parviendraient à dépasser un réel devenu invivable en 
communiant avec une communauté de morts sachant partager leur souffrance. Mais il y a plus. Par le biais de la possession, les perceptions multisensorielles de Yamilka parviennent à canaliser sa douleur, ses désirs et ses rêves. On imagine facilement qu'autrement les épreuves qu'elle a subies, toutes plus cruelles les unes que les autres, l'auraient poussée vers la folie.

29 Au fur et à mesure du tournage, alternativement, Yamilka ne fait plus attention à ma présence, devient ensuite très lucide, puis s'absente mentalement en m'oubliant à nouveau. Yamilka étant allongée dans son lit à la manière d'une odalisque, le dispositif scénique est inhabituel. Il rompt avec les codes de bienséance qui brident d'habitude les relations entre l'interviewer et l'interviewé. Dans cette position, Yamilka ne se présente plus comme une femme forte, comme une puissante médiatrice entre vivants et morts, comme une prêtresse guérisseuse sûre d'elle-même et dominatrice. En s'abandonnant, elle s'imprègne de mots qui la débarrassent des conventions. Elle adopte progressivement une attitude introspective déroulant son histoire intime.

Le scénario s'est nourri progressivement de la relation entre la cinéaste que je suis et le sujet filmé. À partir du moment où l'objectif de la caméra cadre le corps détendu du sujet, l'échange, qui jusqu'à présent reposait exclusivement sur des discussions formelles, est devenu plus complexe. Dans cette mise en scène jouée devant ma caméra, Yamilka révèle des signes montrant qu'elle est en passe de rejoindre l'au-delà. Je cherche alors à me faire la plus discrète possible tandis qu'elle est successivement ellemême, puis incarne Leonardo, son fils Odin et Negro bruto, «son nègre " qui l'appelle, passant du sommeil à un état de transe. Se refléter dans l'autre, devenir l'autre, est loin d'être une expérience anodine. Cette mutation d'identité est l'aboutissement d'un long travail sur soi même. Avant d'être possédé lors de rituels de fêtes ou lors de simples exercices domestiques, il est dans certains cas nécessaire de s'entraîner régulièrement pour parvenir à se détacher du réel et vivre une expérience de fusion avec des êtres fictifs ou réels .

31 Au lieu de tenter de restituer le panthéon et les croyances de Yamilka selon la tradition anthropologique, je me suis ici focalisée sur ses performances, c'est-à-dire sur les expériences mystiques qu'elle vit intensément dans son corps. À l'origine, le départ de son fils Odin et le vide qui a suivi l'ont contrainte à repenser radicalement les relations qu'elles tissent entre vivants et morts. Les apparitions de Negro bruto sont devenues fréquentes au point de ne plus pouvoir s'en passer. Bien que le phénomène se produise quasi quotidiennement, il n'a jamais pris l'aspect d'une routine. Il la transforme chaque fois radicalement.

Qu'est-il arrivé à Yamilka pour en venir à être si intimement liée à un défunt possessif et jaloux? On conviendra que la question de la croyance en Negro bruto est ici secondaire. Le problème n'est pas là. Il faut repartir du constat qu'il se manifeste, qu'il demande à être choyé en recevant sa part de rhum et de cigares, et surtout qu'il veut être aimé. Mais, in fine, qu'est-ce que cela signifie?

Bensa et Fassin relèvent que dans les sociétés où " plus rien ne marche », lorsqu'il y a " rupture de l'intelligibilité », ou encore lorsque des groupes font face à des situations où "rien ne sera plus comme avant", les expériences reposant sur une perception extrasensorielle engageant fortement l'affect des individus tendent à se multiplier ${ }^{9}$. Luc Boltanski fait remarquer de son côté que les apparitions de morts impliquent des moments "méta-ontologiques » dans lesquels la réalité perd de son évidence et doit être reconsidérée ${ }^{10}$. Pour ma part, j'observe que dans un quartier pauvre comme 
Cerrofruto où vit Yamilka, les apparitions des morts sont des "événements " qui structurent la vie sociale. Au plan individuel, Negro bruto aide Yamilka à dépasser ses drames et à établir de solides relations avec la population. Il est intéressant à ce propos de noter qu'elle ne cherche jamais à expliquer la raison des malheurs subis à titre personnel ou subis par d'autres. Elle se contente de chercher à résoudre les situations pour les rendre supportables.

Élisabeth Claverie, qui a étudié les apparitions de la Vierge Marie en BosnieHerzégovine, a souligné que celles-ci consistent en réalité à révéler des drames sociaux habituellement tus. Ainsi la Vierge de Medjugorje apparaît précisément là où ont eu lieu des massacres de masse et où ont été creusées des fosses communes. De même, à Cuba, les morts du Palo monte revivent les blessures profondes de la communauté afrocubaine. Non seulement les morts apparaissent plus fréquemment dans les zones économiquement dévastées, soit dans les quartiers les plus noirs et les plus misérables comme Cerrofruto, mais Negro bruto renvoie à la représentation d'un esclave enchainé et méprisé. Les élites politiques de l'île, descendants pour une large majorité d'entre eux des familles d'esclavagistes européens, restent discrètes sur ce volet douloureux de l'histoire collective en préférant fédérer la population autour de l'internationale communiste. En revanche, Negro bruto, le gnome noir, le gnome laid et puant, se charge de rappeler à la population la plus humble que la réalité présente est dans la continuité des souffrances qu'elle a endurées au temps de l'esclavage. La violence qui l'a durablement meurtrie dans sa chair est aujourd'hui réactivée par les fréquentes apparitions de ses morts.

Dans le film que je suis en train de terminer, je tente d'exprimer la subtilité des émotions vécues par cette population misérable et malgré tout tenue d'être muette sur une douleur séculaire.

\section{BIBLIOGRAPHIE}

BENSA Alban et FASSIN Éric, «Les sciences sociales face à l'événement », dans Terrain, n 38, p. 5-20.

BOLTANSKI Luc, «Institution et critique sociale. Une approche pragmatique de la domination », Tracés, $\mathrm{n}^{\circ}$ 8, p. 7-43.

CLAVERIE Élisabeth, Les guerres de la Vierge. Une anthropologie des apparitions, Paris, Gallimard, 2003.

Descola Philippe, Par-delà nature et culture, Paris, Gallimard, 2005.

Descola Philippe, La fabrique des images, Paris, Ed. Musée du Quai Branly, 2010.

Foucault Michel, «La vie des hommes infâmes ", Les Cahiers du chemin, n 29, 1977, p. 12-29.

HENARE Amiria, HoolBRAD Martin et WASTELl Sari (éd.), Thinking through Things. Theorising Artefacts Ethnographically, Abington-New York, Routledge, 2007

INGOLD Tim, The Perception of the Environment, Essays in livehood, dwelling and skill, Abingdon, Routledge, 2000. 
KERESTEZI Katerina, Vivre avec les morts à Cuba, Paris, Karthala, 2016.

Müller Bernard, Pasqualino Caterina et Schneider Arnd, Le terrain comme mise en scène, Lyon, Presses universitaires de Lyon, 2017.

PASQUALINO Caterina, « Métamorphoses des voix », dans Bertrand Hell et Jean de Loisy (dir), Les Maîtres du désordre, Paris, Éd. Musée du Quai Branly, 2012, p. 202-203.

PASQUALINo Caterina, « Métamorphoses de la voix dans les rites de possession cubains et dans l'art contemporain », Gruppen, $n^{\circ}$ 4, Paris, 2012, p. 126-140.

PASQUALINO Caterina, « Experimental Film, Trance and Near-death Experiences » dans

Experimental film and Anthropology, édité par Caterina Pasqualino et Arnd Schneider, London/New York, Bloomsbury, 2014, p. 45-62.

PASQUALINO Caterina, « Vocalisation of Suffering », dans Suffering, Art and Aesthetic, sous la direction de Ratiba Hadj-Moussa et Michael Nijhawan, Basingstoke, Palgrave Macmillan, 2014, p. 99-120.

PASQUALINO Caterina, « Être chose : marionnettes, possession cubaine et théâtre contemporain ", dans La marionnette : objet d'histoire, oeuvre d'art, objet de civilisation?, sous la direction de Thierry Dufrêne, Paris : Éditions du Cherche midi, 2016.

PASQUALINo Caterina, «L'habitant du chaudron. Se lier aux morts dans le culte Palo Monte » dans Persona Etrangement Humain, dirigé par Emmanuel Grimaud et Anne-Christine Taylor, Éd. Musée du Quai Branly, Paris, 2016.

PASQUALINo Caterina, Tierra Inquieta (avec Chiara Ambrosio), 2017. Film de 75 min. Diffusion: Paris, dans le cycle « Cinéma d'avant garde » dirigé par Nicole Brenez le 17 juin 2016 à la Cinémathèque de Paris ; Athènes, dans Ethnofest (novembre 2018) ; Trento (Italie), dans Film festival (2018).

VIVEIROS DE CASTRo Eduardo, «Cosmological Dexis and Amerindian Perspectivism », Journal of the Royal Anthropological Institute, vol. 4, n 3, 1998, p. 469-488.

\section{NOTES}

1. Suivant les pratiques du Palo monte, petit à petit, Yamilka est devenue une mortera assez reconnue dans son quartier. La mortera est une forme soft du Palo Monte. Le Palo, d'origine bantu, se fonde principallement sur une dévotion aux morts (nfumbi). Le Palero est lié a un mort (il muerto de prenda) qui se matérialise dans un vase rituel (caldero o nganga) constitué d'un assemblage de matières (os, cheveux, clous, bouts de bois, cailloux, terre, etc.) et d'objets (poupée, cadenas, fer à cheval...) hétéroclites concrétisant une relation entre un ici et un au-delà dont la puissance peut d'année en année augmenter.

2. Voir notamment, Philippe Descola, Par-delà nature et culture, Paris, Gallimard, 2005 et La fabrique des images, Paris, Ed. Musée du Quai Branly, 2010.

3. Michel Foucault, «La vie des hommes infâmes», Les Cahiers du chemin, n 29, 1977, p. 12-29.

4. Avec Arnd Schneider et Bernard Müller, j'ai récemment co-édité le livre Le terrain comme mise en scène dans lequel les chercheurs réunis ont tenté de recentrer le propos de leur discipline à partir de la singularité de leur regard et de leurs rencontres, allant jusqu'à reconnaître leurs interlocuteurs comme co-créateurs de leur recherche. Plutôt que de séparer observateur et sujets observés, terrain et travail au bureau, l'anthropologie proposée dans ce livre assume une part de bricolage, laissant apparaître les processus de fabrication, avec leur intensité émotionnelle, les 
doutes théoriques et les errances. Se situant à la croisée de plusieurs pratiques (anthropologie, théâtre, performances d'art contemporain et cinéma), les auteurs admettent que la construction de l'objet de connaissance repose autant sur une vision personnelle que sur le partage d'expériences, assumant parfois leur conception d'un terrain comme utopie collective ou comme partage d'affinités poétiques.

5. L'idée de collaboration est aussi au cœur de mon dernier film Terre inquiète (réalisé avec Chiara Ambrosio), qui met en scène la fiction d'un rituel conçu en collaboration entre les auteurs et des chômeurs de longue durée à Grenade (Espagne). Les protagonistes évoquent leurs difficultés après la crise économique de 2007 et la mémoire encore très présente de la guerre civile espagnole. Leur fierté est d'être parvenus à occuper une décharge illégale et d'avoir transformé cette terre asséchée en un luxuriant jardin potager. Au fil des conversations naît le projet de créer de toutes pièces un " rituel de l'eau ». Les " acteurs " y apparaissent au ralenti et en file indienne, transportant, de la « source des larmes » jusqu'au jardin, de modestes récipients emplis d'eau afin d'apaiser les douleurs de la terre. Alors que cette poignée de militants engagés cherche à restituer le sens de leurs actions à travers une performance théâtrale, j'y interroge les conditions d'émergence de la contagion émotionnelle survenant au sein du groupe.

6. Sur les relations complexes entre les individus et leurs ngangas, lire entre autres Katerina Kerestetzi, Vivre avec les morts à Cuba, Paris, Karthala, 2016.

7. Dans Thinking Through Things, Martin Holbraad analyse le concept de ache: à la fois poudre médicinale essentielle aux fidèles pour mener à bien leurs rituels et puissance spirituelle, pouvoir, grâce.

8. Au cours de mes travaux d'enquête sur les rituels palo monte à Santiago de Cuba (que je présente de manière plus ample dans le projet de recherche), j'ai découvert que la notion de ache peut être comprise comme le déclenchement chez les possédés d'une montée d'émotion exprimée par des frissons. Variantes du célèbre concept polynésien du mana étudié par Mauss, le ache marque une phase d'intensification émotionnelle du rituel. Dans toute possession palo monte, le ache se manifeste lors d'un changement de rythme, lors du solo intempestif d'un participant ou lors d'un mouvement inattendu et brusque du possédé : soit lors d'un «tourbillon dangereux » nommé cruce (dont la traduction littérale est «croisement »). Ces signaux marquent l'arrivée d'un mort venu chasser violemment un autre mort pour s'emparer du corps d'un possédé.

9. Alban Bensa et Eric Fassin, "Les sciences sociales face à l'évènement », dans Terrain, 2002, nº 38 , p. 8

10. Boltanski Luc, «Institution et critique sociale. Une approche pragmatique de la domination », dans Tracés $\mathrm{n}^{\circ} 8$, p. 24.

\section{RÉSUMÉS}

Caterina Pasqualino, réalisatrice et chercheuse, présente sa démarche pour le film qu'elle est en train de terminer. Cuba, par-delà les océans se concentre sur la vie d'une "infâme ", ses souffrances et ses rapports aux rituels. Le film documentaire dont Yamilka est la protagoniste, explore son univers émotionnel et tente de restituer la fluctuation de ses sentiments. L'ex-mari de Yamilka, Leonardo, est parti il y a dix ans de Cuba pour immigrer en France en emmenant avec lui leur fils Odin. Restée sur l'île, Yamilka, la quarantaine, est aujourd'hui devenue une prêtresse mortera, ayant un don de médiation avec les ancêtres défunts pour résoudre les problèmes rencontrés par son entourage. Pour tenter de parer à la douleur provoquée par l'absence de son enfant, elle 
recourt à des séances de possession dans lesquelles l'esprit d'un défunt, Negro bruto, lui permet de le rejoindre par delà les océans.

Caterina Pasqualino, director and researcher, presents her approach for the film she is finishing. Cuba, beyond the oceans focuses on the life of an "infamous", its suffering and its relationship to rituals. The documentary film Yamilla is the main protagonist, explores his emotional world and tries to restore the fluctuation of his feelings.

Yamilka's ex-husband, Leonardo, left Cuba 10 years ago to immigrate to France, taking his son Odin with him. Left on the island, forty years, Yamilka has now become a priestess mortera, having a gift of mediation with the deceased ancestors to solve the problems encountered by those around her. To try to cope with the pain caused by the absence of her child, she resorts to sessions of possession in which the spirit of a deceased, "Negro bruto », allows him to join him across the oceans.

\section{AUTEUR}

\section{CATERINA PASQUALINO}

Ethnologue, chargée de recherche au CNRS (Médaille de bronze en 2000).

Caterina Pasqualino est chargée de recherche au Laboratoire d'Anthropologie des Institutions et des Organisations Sociales, à Paris. Également réalisatrice de films documentaires, ses recherches récentes l'amènent à s'interroger sur la notion de performance rapportée à la voix et à la gestuelle (chant, danse) comme enjeu identitaire et politique.

Après avoir étudié plusieurs minorités du bassin méditerranéen, elle entreprend un travail sur les populations gitanes. Son second ouvrage, Dire le chant. Les Gitans flamencos d'Andalousie (CNRS édition, Paris, 1998), constitue la première étude monographique des Gitans. Elle innove en abordant, dans une perspective anthropologique, le flamenco andalou, genre musical habituellement entrevu en terme pittoresque ou musicologique. 